\title{
3D Model-Based Simulation Analysis of Energy Consumption in Hot Air Drying of Corn Kernels
}

\author{
Shiwei Zhang, Ninghua Kong, Yufang Zhu, Zhijun Zhang, and Chenghai Xu \\ School of Mechanical Engineering and Automation, Northeastern University, P.O. Box 319, Shenyang 110004, China \\ Correspondence should be addressed to Shiwei Zhang; shwzhang@mail.neu.edu.cn
}

Received 11 March 2013; Accepted 9 May 2013

Academic Editor: Hua-Shu Dou

Copyright (C) 2013 Shiwei Zhang et al. This is an open access article distributed under the Creative Commons Attribution License, which permits unrestricted use, distribution, and reproduction in any medium, provided the original work is properly cited.

\begin{abstract}
To determine the mechanism of energy consumption in hot air drying, we simulate the interior heat and mass transfer processes that occur during the hot air drying for a single corn grain. The simulations are based on a 3D solid model. The 3D real body model is obtained by scanning the corn kernels with a high-precision medical CT machine. The CT images are then edited by MIMICS and ANSYS software to reconstruct the three-dimensional real body model of a corn kernel. The Fourier heat conduction equation, the Fick diffusion equation, the heat transfer coefficient, and the mass diffusion coefficient are chosen as the governing equations of the theoretical dry model. The calculation software, COMSOL Multiphysics, is used to complete the simulation calculation. The influence of air temperature and velocity on the heat and mass transfer processes is discussed. Results show that mass transfer dominates during the hot air drying of corn grains. Air temperature and velocity are chosen primarily in consideration of mass transfer effects. A low velocity leads to less energy consumption.
\end{abstract}

\section{Introduction}

Hot air drying is a commonly used method for drying corn; it is often characterized by high energy consumption and low efficiency [1]. Many studies have been conducted on the drying characteristics and energy consumed when drying thin layers of vegetables, fruits, and food [2,3]. These studies usually focus on the optimization of drying systems and drying methods. To date, no research has been devoted to the drying characteristics and energy consumed during the hot drying of a single corn kernel even though the drying process of each single grain is the theoretical basis of various drying techniques. In the current work, we investigate the drying characteristic of single corn kernels and provide theoretical support for reducing energy consumption in hot air drying.

Given that the kernel structures and shapes of grains are neither uniform nor regular, describing their drying processes on the basis of the original shape of a single grain kernel is difficult to accomplish. Most of the previously conducted simulations of material drying, especially for singlebody corn kernels, are based on ideal models that are established by simplifying the shapes of dried materials. Shape simplification enables easy simulation calculation but causes original shape-related errors in the simulations. Gustafson simplified a grain kernel into a 2D irregular shape on the basis of original grain size and then simulated the drying process by finite element method (FEM) [4]. Li and Cao simplified a single rough rice kernel as spherical to simulate moisture diffusion with a three-layer body model [5]. Zhang and Kong studied the heat and mass transfer of porous medium in the vacuum drying process by using a $1 \mathrm{D}$ model [6]. Jia and Cao simplified an irregular corn particle into a 2D irregular geometric model to analyze heat and mass transfer in corn dying by FEM [7]. Neményi and Czaba established a finite element model to investigate the heat and moisture distributions in a cross-sectional area of an individual maize kernel [8].

Grain kernel simulation based on a 3D real body model has recently become a trend in simulation studies because it generates more accurate results. The simulation of the heat and mass transfer in corn kernels with a 3D model has been carried out [9]. Ghosh et al. obtained a 3D geometric model of a wheat kernel by magnetic resonance imaging (MRI) and developed a mathematical model for moisture diffusion in 


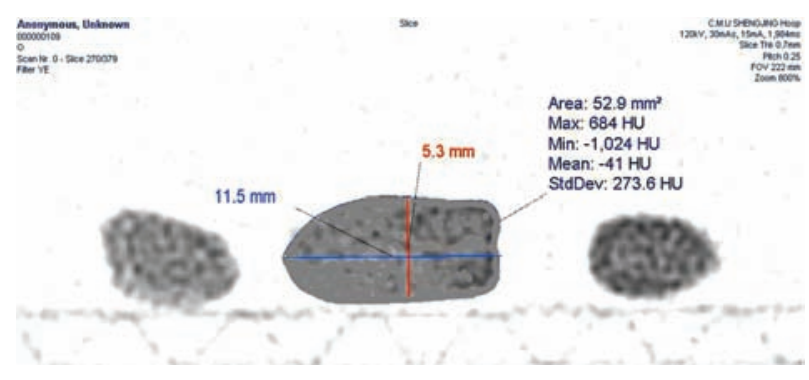

FIGURE 1: CT slice images of the corn grain.

a single wheat kernel during the drying process [10]. Perez et al. constructed a 3D model with tomographic images and simulated the mass transfer and hygroscopic cracks in rice grains [11]. The present work is carried out on the basis of the aforementioned studies.

\section{Real Body Model}

2.1. Review of Real Body Model Reconstruction. Grain kernels are usually simplified into spherical, cylindrical, ellipsoidal, and flat chip shapes because the shapes of grain particle shapes are irregular and variable. Given that a simplified model cannot reflect solid features, obtaining a more accurate model facilitates the understanding of the heat and mass transfer mechanism in grain kernels. MRI has recently been used to obtain 3D real body models. Song developed a $3 \mathrm{D}$ microscopic MRI with the $3 \mathrm{D}$ projection reconstruction (3DPR) technique to measure the moisture distribution in a single mature wheat grain [12]. Goñi obtained the slices of irregularly shaped food with nonuniform rational B-splines and MRI techniques and reconstructed a 3D geometric model [13]. Ghosh et al. constructed a 3D geometric model of wheat kernel by MRI [10], and Perez et al. built a 3D model with tomographic images [11]. Obtaining 3D geometric models by MRI presents high cost and complexity, driving the identification of a more convenient method. The CT scanning technique based on $3 \mathrm{D}$ medical image reconstruction is extensively used in the field of medicine. In this paper, the CT scanning technology is used to create a $3 \mathrm{D}$ real body model of a single corn kernel.

2.2. Steps to Obtain the 3D Model. The steps for creating the $3 \mathrm{D}$ real body model are as follows. (1) Several selected corn grains are scanned by high-precision medical CT to obtain serried transect image data. (2) The image data is treated by MIMICS software in turn. (3) The contour line image is transferred into ANSYS software and converted into a 3D real body model, which is nearly of the same shape as the original corn grain.

2.2.1. Scanning with CT. Scanning pertains to the use of computer technology for the tomographic reconstruction of a measured object into a 3D topographic image. Single axis Xrays are projected onto the object to be tested. On the basis of the difference in radiation absorption rate and transmissivity among the tested objects, a computer collects data from the rays and reconstructs 3D images. This study uses the Philips Brilliance iCT 256 layer spiral quick CT at Shengjing Hospital, an affiliated hospital of the Chinese Medical University. Corn grains are scanned to obtain high-precision CT images, with a scanning thickness of $0.625 \mathrm{~mm}$. The CT image files are stored in Digital Imaging and Communication in Medicine (Figure 1).

2.2.2. Image Treatment in MIMICS. MIMICS is a set of highly integrated software for generating, editing, and treating $3 \mathrm{D}$ images. It can accept various types of original scanning data (from CT or MRI), set up corresponding 3D models, and output these in general CAD format. In this study, MIMICS software is used to treat CT image data of corn kernels to generate a $3 \mathrm{D}$ geometric contour model of a corn grain, to modify parts of the contour model for surface smoothness, and to transmit the contour line image model to ANSYS software.

(a) The CT images are input into MIMICS, and one corn kernel area is chosen, with adjustments on the threshold between -228 and 2872 Hounsfield. The images are edited until a clear and intact contour is generated.

(b) The "Crop Mash" function is used to divide contour regions, the "Calculate Polylines" function is used to generate the 3D geometric contour model of a corn grain, and the surface contour model is smoothed with the Magices 9.9.

(c) The surface contour model is exported as a file in an "ANSYS area file" (.lis) format.

2.2.3. Image Treatment in ANSYS. The contour model is converted into a $3 \mathrm{D}$ real body model, which has nearly the same shape as the original corn grain. The $3 \mathrm{D}$ real body model of a corn grain is generated in ANSYS and exported as an "Initial Graphics Exchange Specification" (IGES) file for postprocessing in COMSOL software.

The 3D real body model obtained through the aforementioned method is more accurate than other simplified models [4-8]. This kind of reconstruction method is more convenient and practical. 

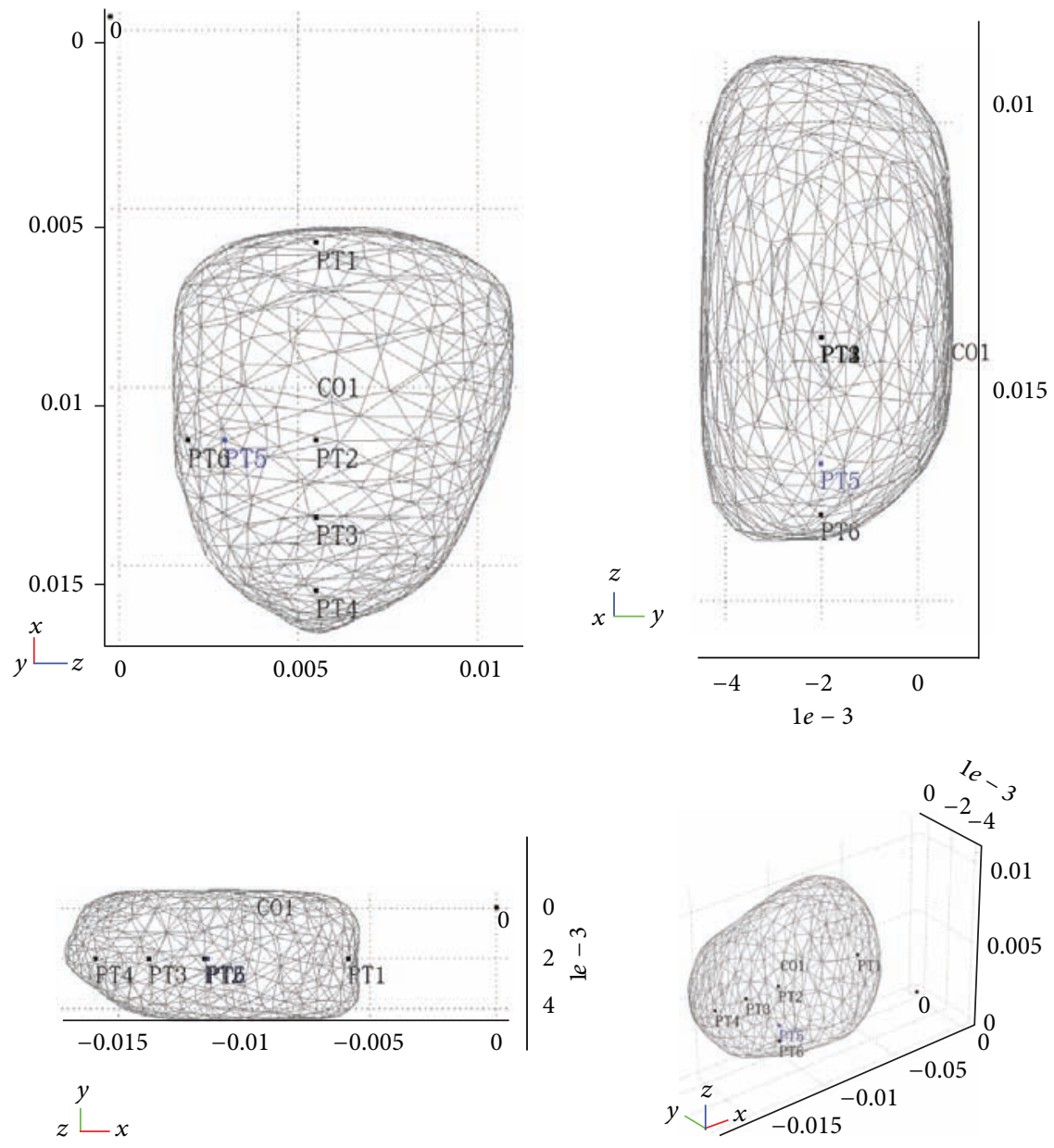

FIgURE 2: 3D real body model in COMSOL and locations of detection points.

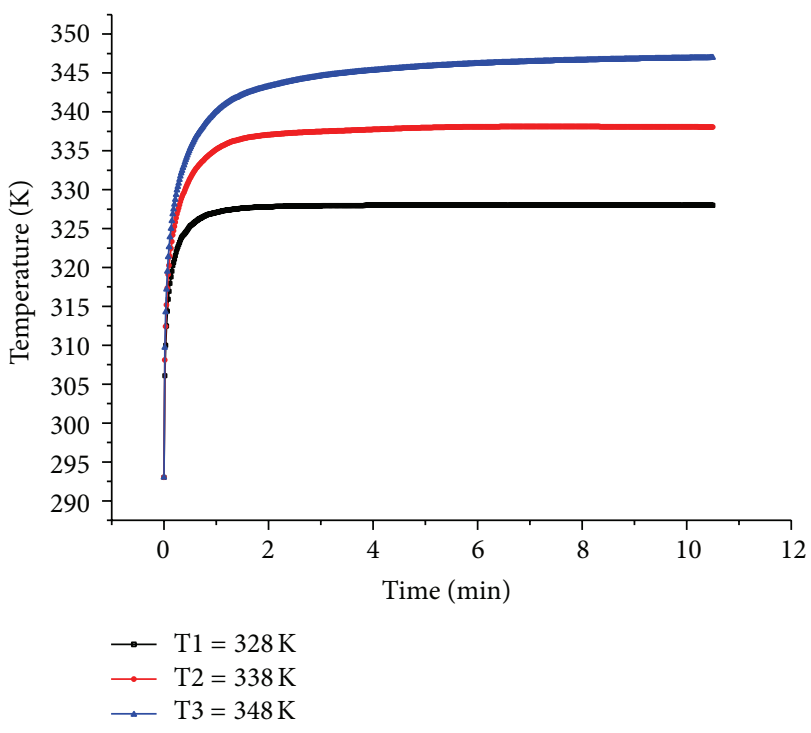

FIGURE 3: Average temperature curves of the corn kernel at different drying temperatures.

\section{Mathematical Model}

3.1. Hypothesis. For this study, we make the following assumptions. (1) The internal temperature and moisture of corn

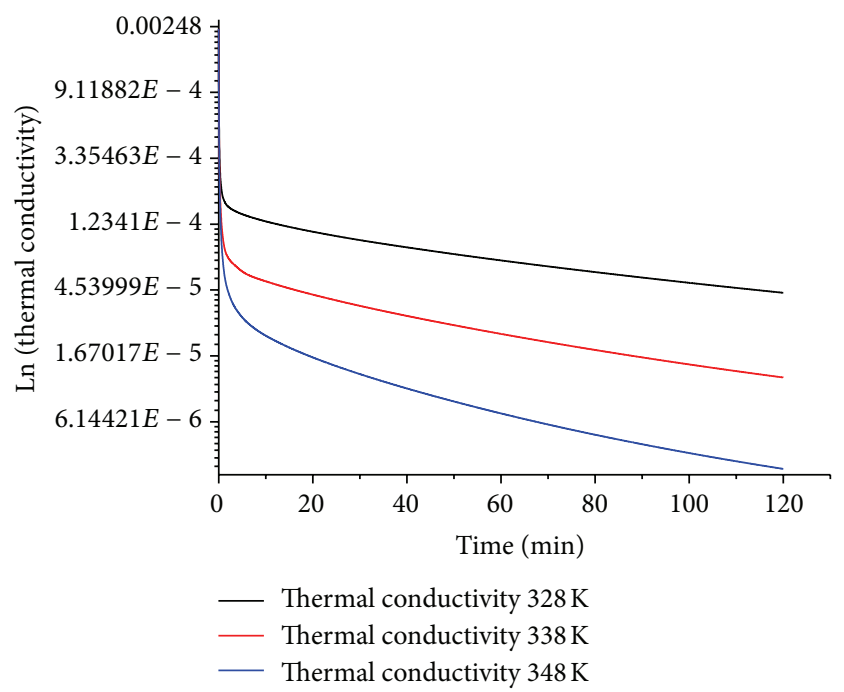

FIGURE 4: Thermal conductivity curves at different drying temperatures.

are evenly distributed at the beginning of drying process. (2) The changes in shrinkage and shape of the corn grain are disregarded during the drying process. (3) The various components of corn grain are isotropic and uniform. (4) Water 


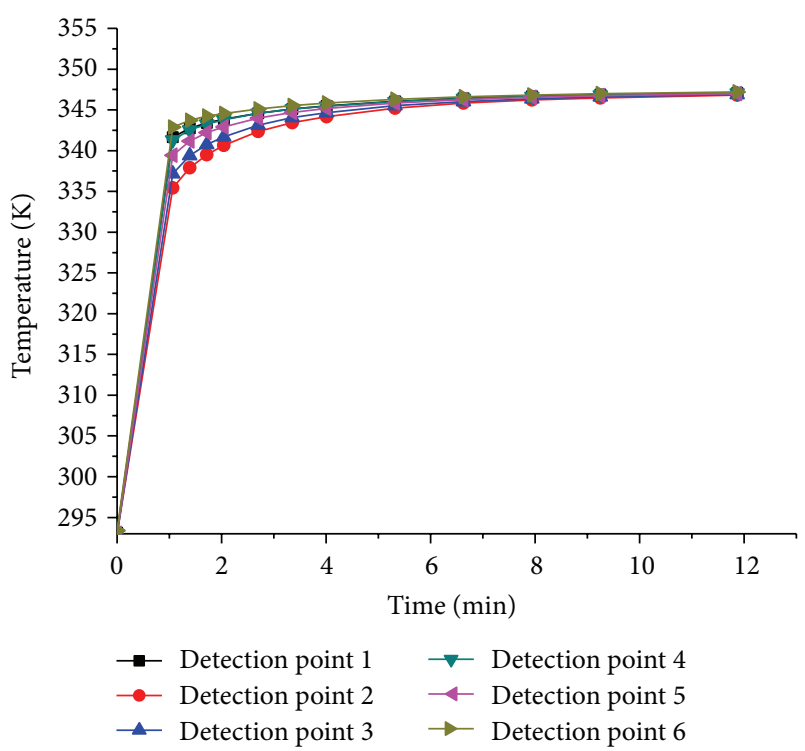

FIgURE 5: Temperature curves at detection points when $T_{3}=348 \mathrm{~K}$.

is diffused to the external boundary, and water vaporization occurs on the grain surface.

3.2. Heat Transfer Equation. The heat transfer in the grain kernels is modeled following Fourier's law of heat conduction and the first law of thermodynamics [14]. Heat $Q_{i}(i=x, y, z)$ is generated by any microunit at a given position. Heat $Q_{t}$ is generated by any microunit at a given time. Heat $Q_{H}$ is conserved or released by changes in the temperature and material of a microunit:

$$
\begin{gathered}
Q_{x}=\frac{\partial}{\partial x}\left(\lambda \frac{\partial T}{\partial x}\right) d x d y d z \\
Q_{y}=\frac{\partial}{\partial y}\left(\lambda \frac{\partial T}{\partial y}\right) d x d y d z \\
Q_{z}=\frac{\partial}{\partial z}\left(\lambda \frac{\partial T}{\partial z}\right) d x d y d z \\
Q_{t}=q_{v} d x d y d z \\
Q_{x}+Q_{y}+Q_{z}+Q_{t}=Q_{H}, \\
\frac{\partial}{\partial x}\left(\lambda \frac{\partial T}{\partial x}\right)+\frac{\partial}{\partial y}\left(\lambda \frac{\partial T}{\partial y}\right)+\frac{\partial}{\partial z}\left(\lambda \frac{\partial T}{\partial z}\right)+q_{v}=\frac{\partial}{\partial t}(\rho H) .
\end{gathered}
$$

If moisture vaporizes in the kernel,

$$
\begin{gathered}
H=h_{f g} M+c_{p} T, \\
\frac{\partial H}{\partial t}=h_{f g} \frac{\partial M}{\partial t}+c_{p} \frac{\partial T}{\partial t} .
\end{gathered}
$$

The 3D mathematical model of heat transfer is

$$
\frac{\partial}{\partial x}\left(\lambda \frac{\partial T}{\partial x}\right)+\frac{\partial}{\partial y}\left(\lambda \frac{\partial T}{\partial y}\right)+\frac{\partial}{\partial z}\left(\lambda \frac{\partial T}{\partial z}\right)+q_{v}
$$

$$
=\rho c_{p} \frac{\partial T}{\partial t}+\rho h_{f g} \frac{\partial M}{\partial t}
$$

where $x, y$, and $z$ are the Cartesian coordinates that represent the corn kernel as a $3 \mathrm{D}$ body; $q_{v}$ is the heat generating rate of the inner heat source $\left(\mathrm{J} \mathrm{m}^{-3} \mathrm{~s}^{-1}\right) ; \rho$ is the density of corn $\left(\mathrm{kg} \mathrm{m}^{-3}\right) ; c_{p}$ denotes the specific heat of corn $\left(\mathrm{J} \mathrm{kg}^{-1} \mathrm{~K}^{-1}\right) ; \lambda$ represents the thermal conductivity of corn $\left(\mathrm{W} \mathrm{m}^{-1} \mathrm{~K}^{-1}\right) ; M$ is the moisture content (dry base); $T$ is the temperature of the corn kernel $(\mathrm{K}) ; h_{f g}$ is the latent heat of water vaporization $\left(\mathrm{J} \mathrm{kg}^{-1}\right)$.

The initial condition is

$$
\left.T\right|_{t=0}=T_{0} .
$$

The boundary condition is

$$
-\lambda\left(\frac{\partial T}{\partial x}+\frac{\partial T}{\partial y}+\frac{\partial T}{\partial z}\right)=h_{t}\left(T-T_{a}\right),
$$

where $h_{t}$ is the convective heat transfer coefficient $\left(\mathrm{W} \mathrm{m}^{-2}\right.$ $\mathrm{K}^{-1}$ ) and $T_{a}$ is the temperature of the drying medium (K).

3.3. Mass Transfer Equation. The moisture diffusion differential equation is modeled following Fick's law [14]. The 3D mathematical model of mass diffusion is expressed as

$$
\frac{\partial M}{\partial t}=D \frac{\partial^{2} M}{\partial x^{2}}+D \frac{\partial^{2} M}{\partial y^{2}}+D \frac{\partial^{2} M}{\partial z^{2}},
$$

where $D$ is the diffusion coefficient $\left(\mathrm{m}^{2} \mathrm{~s}^{-1}\right)$.

The initial condition is

$$
\left.M\right|_{t=0}=M_{0} .
$$

The boundary condition is

$$
-D\left(\frac{\partial M}{\partial x}+\frac{\partial M}{\partial y}+\frac{\partial M}{\partial z}\right)=h_{m}\left(M-M_{e}\right),
$$

where $h_{m}$ is the surface mass transfer coefficient $\left(\mathrm{m} \mathrm{s}^{-1}\right)$ and $M_{e}$ is the equilibrium moisture content (d.b.).

3.4. Energy Consumption Analysis. The energy consumption in the hot air drying process is due primarily to the velocity and temperature of hot wind, as determined by dimensional analysis. The energy consumption dimension is $W \mathrm{~J} /\left(\mathrm{m}^{2} \mathrm{~s}\right)$; the dimension of hot wind velocity $u$ is $\mathrm{m} / \mathrm{s}$; the dimension of hot wind temperature $T$ is $\mathrm{K}$. The air mass flow is $G=$ $u \rho_{a}$ expressed in units of $\mathrm{kg} /\left(\mathrm{m}^{2} \mathrm{~s}\right)$. In accordance with the principle of dimensional homogeneity, density $\rho\left(\mathrm{kg} / \mathrm{m}^{3}\right)$ and specific heat $C_{p} \mathrm{~J} /(\mathrm{kg} \mathrm{K})$ are introduced to establish the equation. We can obtain the energy consumption per unit area; thus,

$$
W=\phi \Delta T C_{p} G=\phi \rho_{a} \Delta T C_{p} u \mathrm{~J} /\left(\mathrm{m}^{2} \mathrm{~s}\right),
$$

where $\phi$ is constant. 

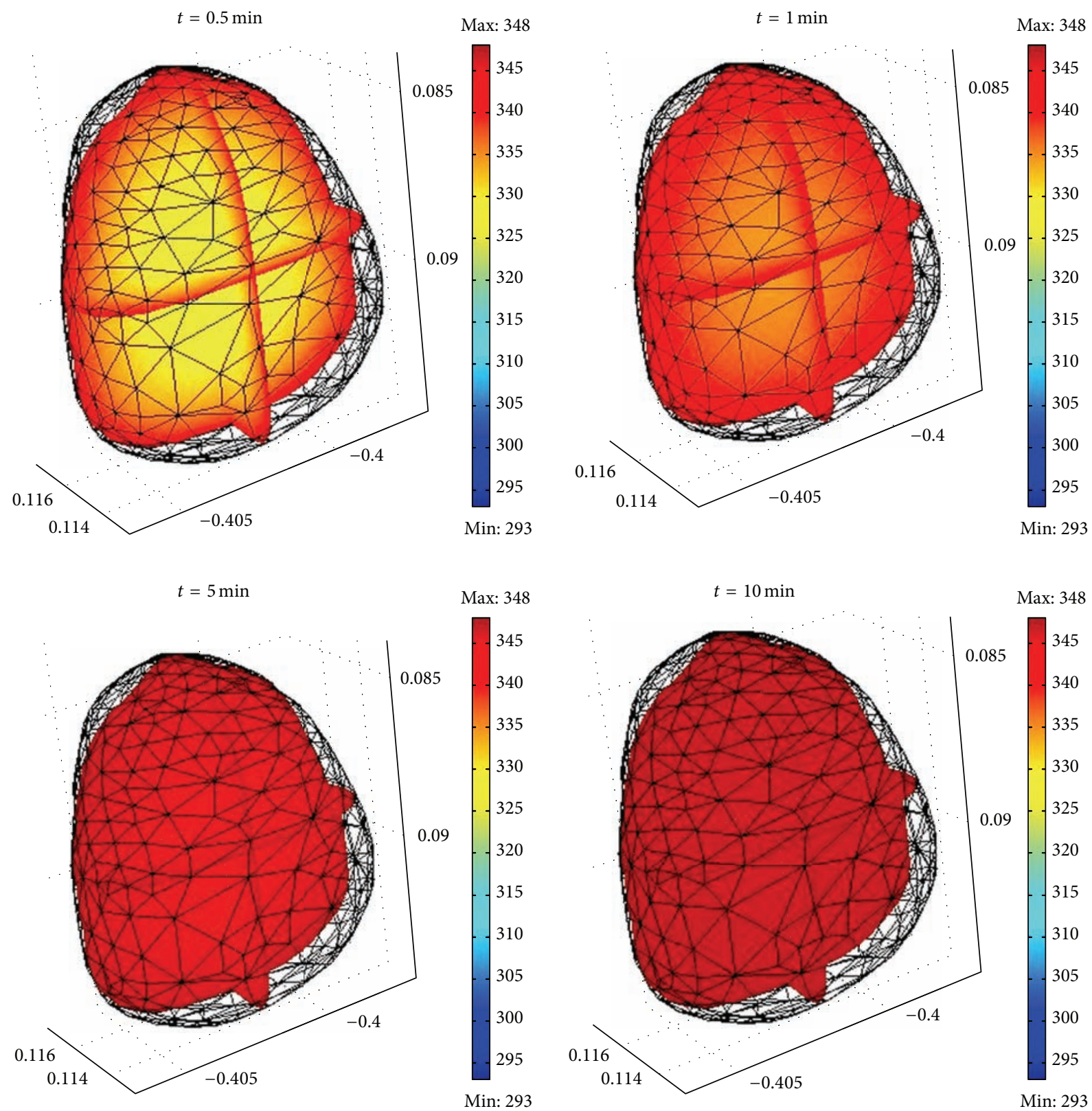

FIGURE 6: Slices of temperature distribution in the corn kernel at different periods.

3.5. Physical and Material Properties. The initial moisture is $M=0.23$ (d.b.); the initial temperature is $T_{0}=293 \mathrm{~K}$; the drying temperatures are $T_{1}=328 \mathrm{~K}, T_{2}=338 \mathrm{~K}$, and $T_{3}=348 \mathrm{~K}$; the hot air velocity is $u_{0}=1.64 \mathrm{~m} / \mathrm{s}$ [7]. The density of the corn kernel [8] is uniform at $\rho=1150 \mathrm{~kg} \mathrm{~m}^{-3}$; the density of air is $\rho_{a}=1.29 \mathrm{~kg} \mathrm{~m}^{-3}$. The specific heat due to the moisture content of the germ and endosperm is expressed as

$$
\begin{gathered}
c_{p 1}=3379 M+1433, \\
c_{p 2}=2565 M+1272.7 .
\end{gathered}
$$

The average specific heat [8] is $c_{p 2}=2000 \mathrm{~J} /(\mathrm{kg} \mathrm{K})$; the latent heat of water vaporization [8] is $h_{f g}=2256267 \mathrm{~J} \mathrm{~kg}^{-1}$; the surface mass transfer coefficient [15] is $h_{m}=0.05 \mathrm{~m} \mathrm{~s}^{-1}$.
The surface heat transfer coefficient [14] is

$$
h_{t}=100 G^{0.49} \mathrm{~W} /\left(\mathrm{m}^{2} \mathrm{~K}\right) \text {. }
$$

The thermal conductivity [7] is

$$
\begin{gathered}
\lambda=\exp \left(-1.74-3.7 M+4.72 \times 10^{-3} T+6.48 M^{2}\right. \\
\left.-1.5 \times 10^{-4} T^{2}+6.27 \times 10^{-2} M T\right) .
\end{gathered}
$$

The diffusion coefficient [16] is

$$
D=a_{0} \exp \left(a_{1} M\right) \exp \left(-\frac{a_{2}}{T}\right)
$$


where $a_{0}, a_{1}, a_{2}$ are constants; $a_{0}=7.817 \times 10^{-5} ; a_{1}=5.5$; $a_{2}=4850$.

\section{Solution via COMSOL Multiphysics}

The calculation process for the simulation is implemented with COMSOL Multiphysics 3.5a. The "diffusion module" and "heat transfer module" in COMSOL software are chosen to simulate the moisture migration and heat transfer process in the corn kernel during the hot air drying process. One of the models is shown in Figure 2. The point $\mathrm{O}$ is the origin point of the coordinate system in the model, the number of free grids is 14440 , the volume is $3.22701 \mathrm{e}^{-7} \mathrm{~m}^{3}$, and six detection points (1-6) are selected from different locations (Table 1). These points are the representative positions that show the calculation results. The points 1 and 6 are the detection points between the cortical layer and endosperm. The point 2 is the detection point at the inner endosperm. The points 3 and 5 are the detection points at the germ. The point 4 is the detection at the radicle.

After the material properties are defined, the governing equations and initial conditions for the inner component of the model and the boundary conditions on the surface of the model are established. The $3 \mathrm{D}$ real body model is now ready for simulation calculation. The mathematical calculation model, which includes the governing equation of heat and mass transfer and the defining equations of property parameters, is presented in the succeeding section. The simulation can be completed by loop computation.

\section{Results and Discussion}

5.1. Influence of Air Temperature on Heat Transfer. In the simulation, the hot wind temperature changes from $T_{1}$ to $T_{3}$ $\left(T_{1}=328 \mathrm{~K}, T_{2}=338 \mathrm{~K}\right.$, and $\left.T_{3}=348 \mathrm{~K}\right)$. Figure 3 shows that the temperature of the corn kernel exhibits increasing curves during the drying process. The increasing trends of temperature are similar. They all reach equilibrium within 10 minutes. The higher the hot wind temperature is, the faster the temperature increases. However, it takes a little more time to reach a balanced drying temperature at a high hot wind temperature than at low hot wind temperature.

The comparison of the thermal conductivity logarithmic $(\ln \lambda)$ curves is shown in Figure 4 . The figure shows the differences in heat transfer at different drying temperatures. As indicated in (12), thermal conductivity changes with temperature during the drying process. The higher the drying temperature, the lower the thermal conductivity.

The temperature at the detection points shows that the corn temperature increases to the equilibrium level in 8 minutes (Figures 5 and 6). The temperature increase at the points near the boundary proceeds at a faster rate than does the temperature increase at the internal points; the temperature distribution in the corn kernel also shows the short-term temperature changes (Figure 6). Figure 6 shows the temperature distribution in the corn kernel at $t=0.5 \mathrm{~min}$, $t=1 \mathrm{~min}, t=5 \mathrm{~min}$, and $t=10 \mathrm{~min}$. The heat transfer rapidly occurs in the hot air drying process. The resistance to
TABLE 1: Locations of detection points.

\begin{tabular}{lc}
\hline Point number & Location \\
\hline 1 & $(-0.006,-0.002,0.0055)$ \\
2 & $(-0.0114,-0.002,0.0055)$ \\
3 & $(-0.0135,-0.002,0.0055)$ \\
4 & $(-0.0155,-0.002,0.0055)$ \\
5 & $(-0.0114,-0.0025,0.003)$ \\
6 & $(-0.0114,-0.002,0.002)$ \\
\hline
\end{tabular}

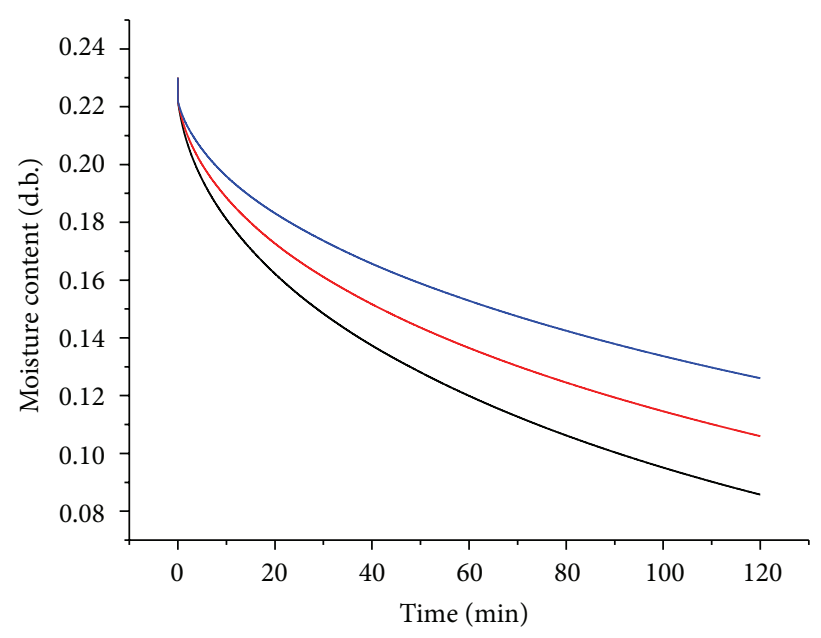

- Average moisture content, $348 \mathrm{~K}$

- Average moisture content, $338 \mathrm{~K}$

_ Average moisture content, $328 \mathrm{~K}$

Figure 7: Drying curves (d.b.) at different temperatures.

heat transfer in the corn kernel is weak [9]. The influence of temperature change on heat transfer is limited, and the effects occur only at the beginning of the drying process.

\subsection{Influence of Air Temperature on Mass Transfer. Figure 7} shows the moisture drying curves at different temperatures. At a drying temperature $T_{3}=348 \mathrm{~K}$, moisture diffuses at the fastest rate. The higher the temperature is, the faster the moisture diffuses.

Representative model-derived images of the kernel slices are shown in Figures 8, 9, and 10. They show the moisture distribution in the corn kernel at $t=10 \mathrm{~min}, t=30 \mathrm{~min}$, $t=60 \mathrm{~min}$, and $t=120 \mathrm{~min}$ at drying temperatures of $T_{1}=328 \mathrm{~K}, T_{2}=338 \mathrm{~K}$, and $T_{3}=348 \mathrm{~K}$. The different colors correspond to the special values of moisture content. The indication of moisture migration at different locations is visually represented with the $3 \mathrm{D}$ body model. The moisture content at $t=60 \mathrm{~min}$ at a drying temperature of $T_{3}=348 \mathrm{~K}$ is nearly the same as that at $t=120 \mathrm{~min}$ at a drying temperature of $T_{1}=328 \mathrm{~K}$.

Diffusion coefficient $D$ changes with temperature and moisture content, as indicated in (11). The average diffusion coefficient curves at different drying temperatures are shown in Figure 11. Diffusion coefficient $D$ rapidly increases at the beginning of drying until it reaches the peak value. It then 

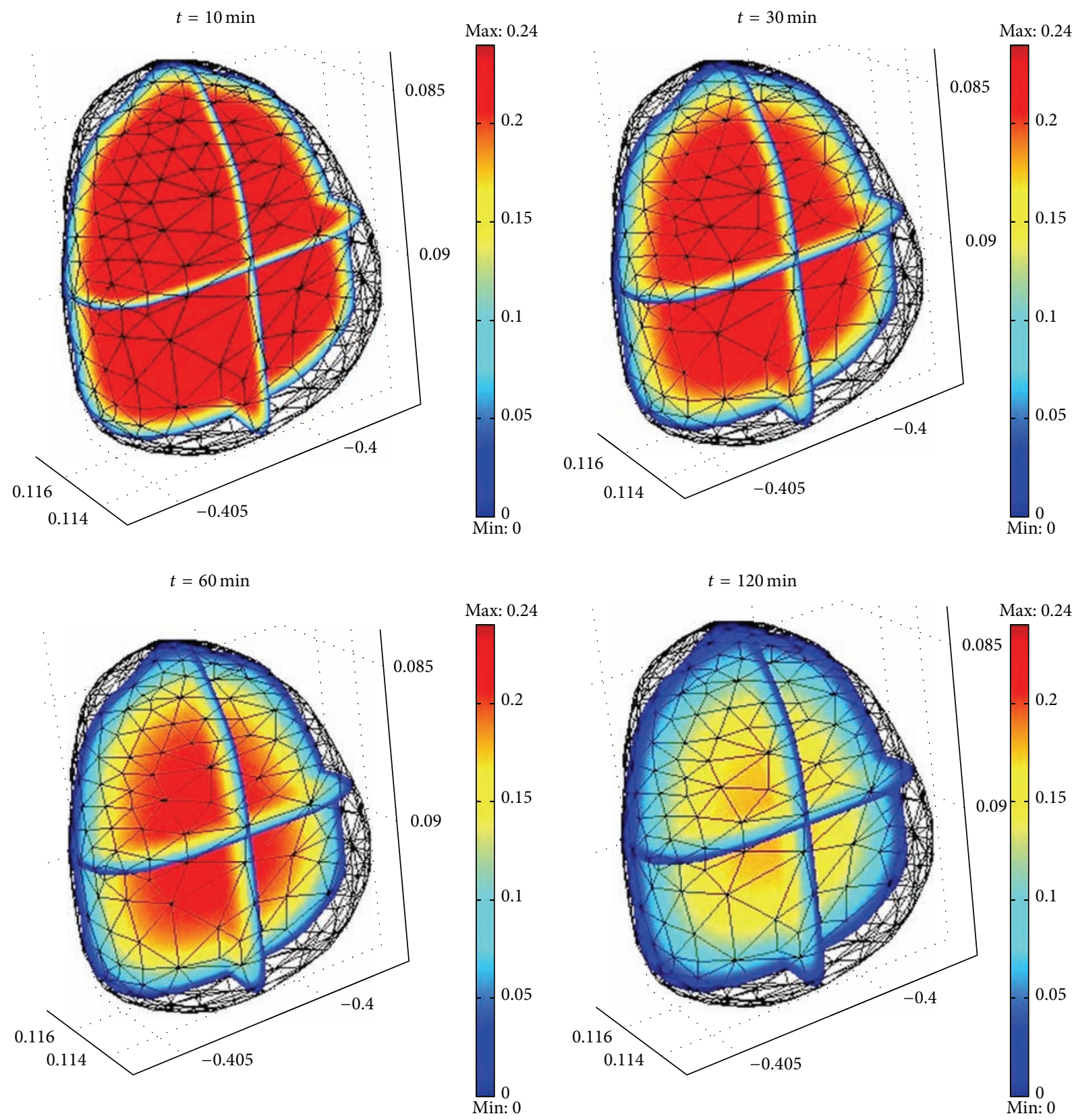

FIGURE 8: Slices of moisture distribution in the corn kernel at drying temperature $T_{1}=348 \mathrm{~K}$.

approaches the equilibrium value. The value of the diffusion coefficient at $T_{3}=348 \mathrm{~K}$ is nearly two times higher than its value at $T_{1}=328 \mathrm{~K}$. The equilibrium diffusion coefficient at drying temperature $T_{3}=348 \mathrm{~K}$ is higher than that at a drying temperature $T_{1}=328 \mathrm{~K}$. The variation range at drying temperature $T_{3}$ between the peak value and equilibrium value is larger than that at $T_{1}=328 \mathrm{~K}$. The higher the drying temperature selected, the larger the diffusion coefficient.

The mass diffusion coefficient visibly changes with temperature before the temperature of the corn kernel reaches the equilibrium temperature (Figure 12). The temperature increase occurs within a short period at the beginning of drying (Figure 5). Temperature visibly influences mass transfer at the beginning of the drying process. Selecting a reasonable drying temperature is necessary for improving drying efficiency and reducing energy consumption.

The moisture distribution in the corn kernel is nonuniform because of the unique organization of the corn kernel structure. Figure 13 shows the drying curves of different detection points (defined in Figure 2) in the corn kernel. The diffusion coefficient curves at different detection points in the drying process are shown in Figure 14. The closer the detection points are to the boundaries, the more easily moisture content is diffused.

The comparison of Figures 13 and 14 shows that the trend of the mass diffusion coefficient curves is similar to that of 

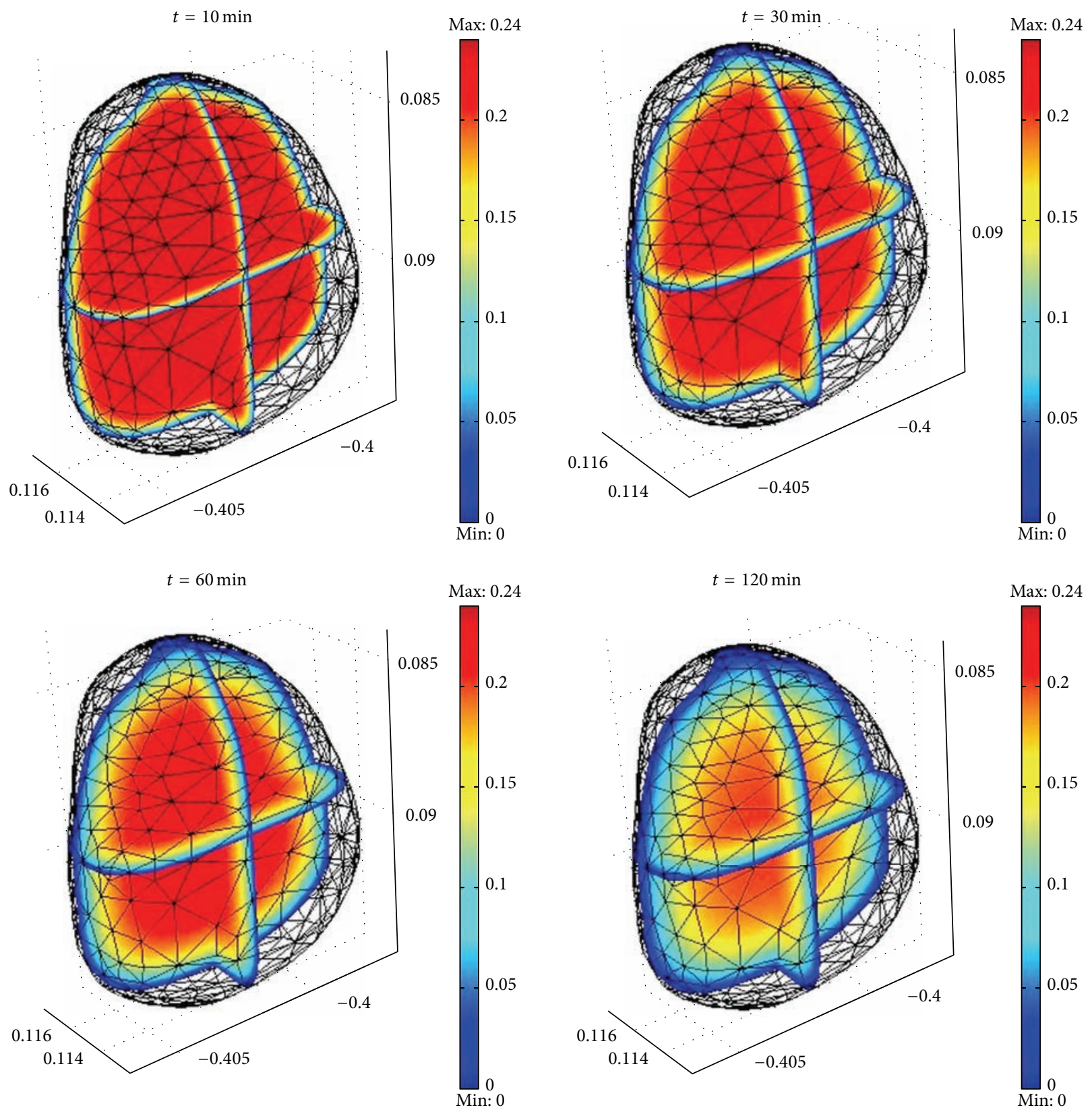

FIGURE 9: Slices of moisture distribution in the corn kernel at drying temperature $T_{2}=338 \mathrm{~K}$.

the moisture content curves. We can deduce that the diffusion coefficient is influenced primarily by moisture content after temperature reaches the equilibrium drying temperature. Mass transfer resistance plays an important role in the drying process after the temperature reaches the equilibrium value.

5.3. Influence of Air Velocity on Hot Air Drying. Hot wind velocity influences energy consumption during hot air drying. In the simulation, the comparisons are processed at $u_{1}=$ $u_{0}, u_{2}=u_{0} / 2$, and $u_{3}=u_{0} / 10$. The temperature and drying curves change with hot wind velocity at the same air temperature (Figures 15 and 16), indicating that effects on heat and mass transfer could be disregarded. When hot wind velocity changes from $u_{1}$ to $u_{2}$, almost no change occurs.

Figure 15 also shows the comparison between simulation drying curves and experiment drying curve (d.b.). In the experiment, the Ohaus MB45 Halogen Moisture Analyzer is used as a hot air dryer. The initial moisture content of corn kernel is 0.23 (d.b). The condition of experiment is hot wind velocity $u_{1}=u_{0}$ and $T=348 \mathrm{~K}$. The experimental drying curve and the simulation curve change with the same trend.

Hot wind velocity directly influences the heat surface transfer coefficient, as shown in (11). The higher the hot wind velocity, the larger the surface transfer coefficient (Figure 17). 

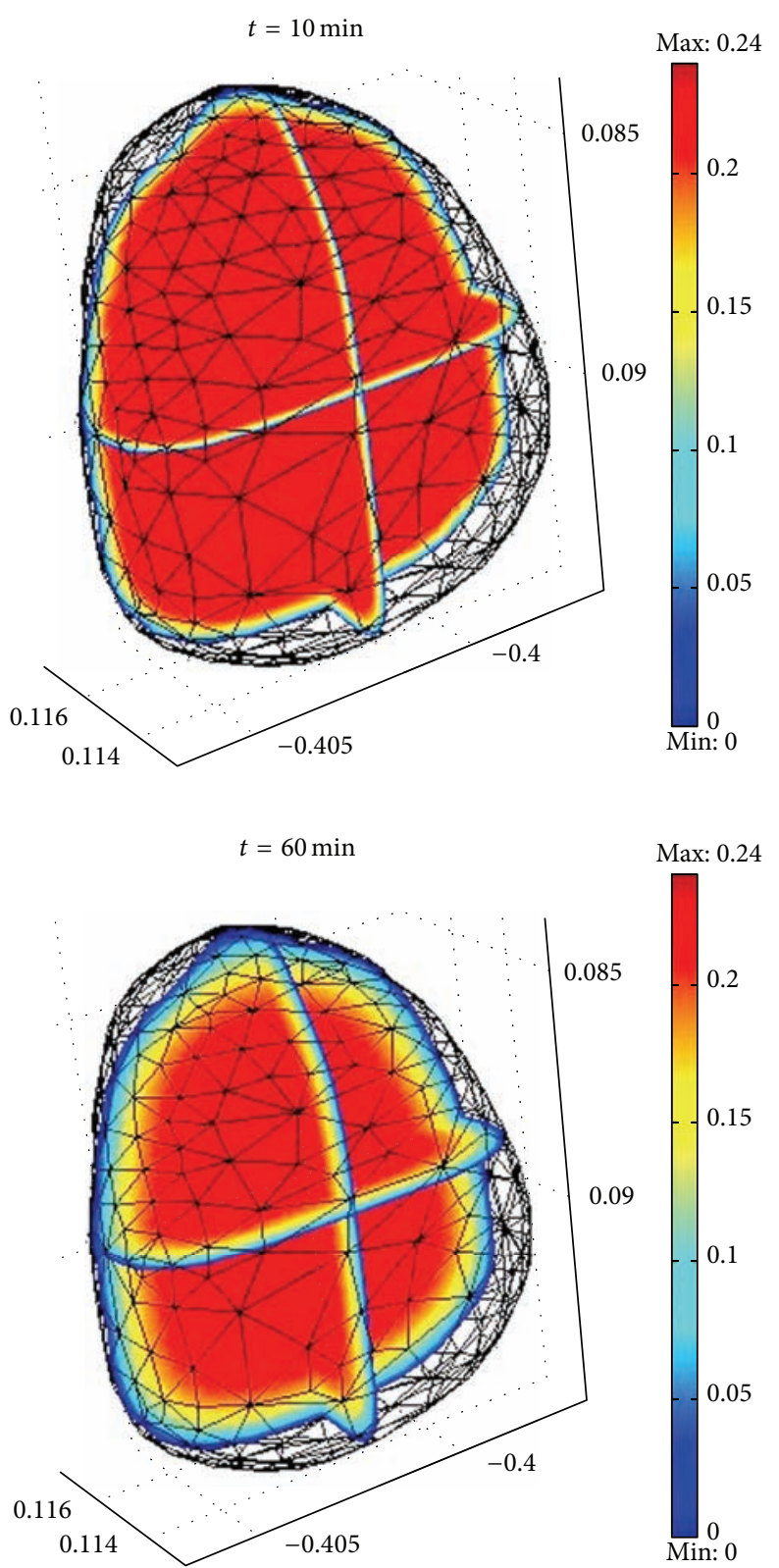
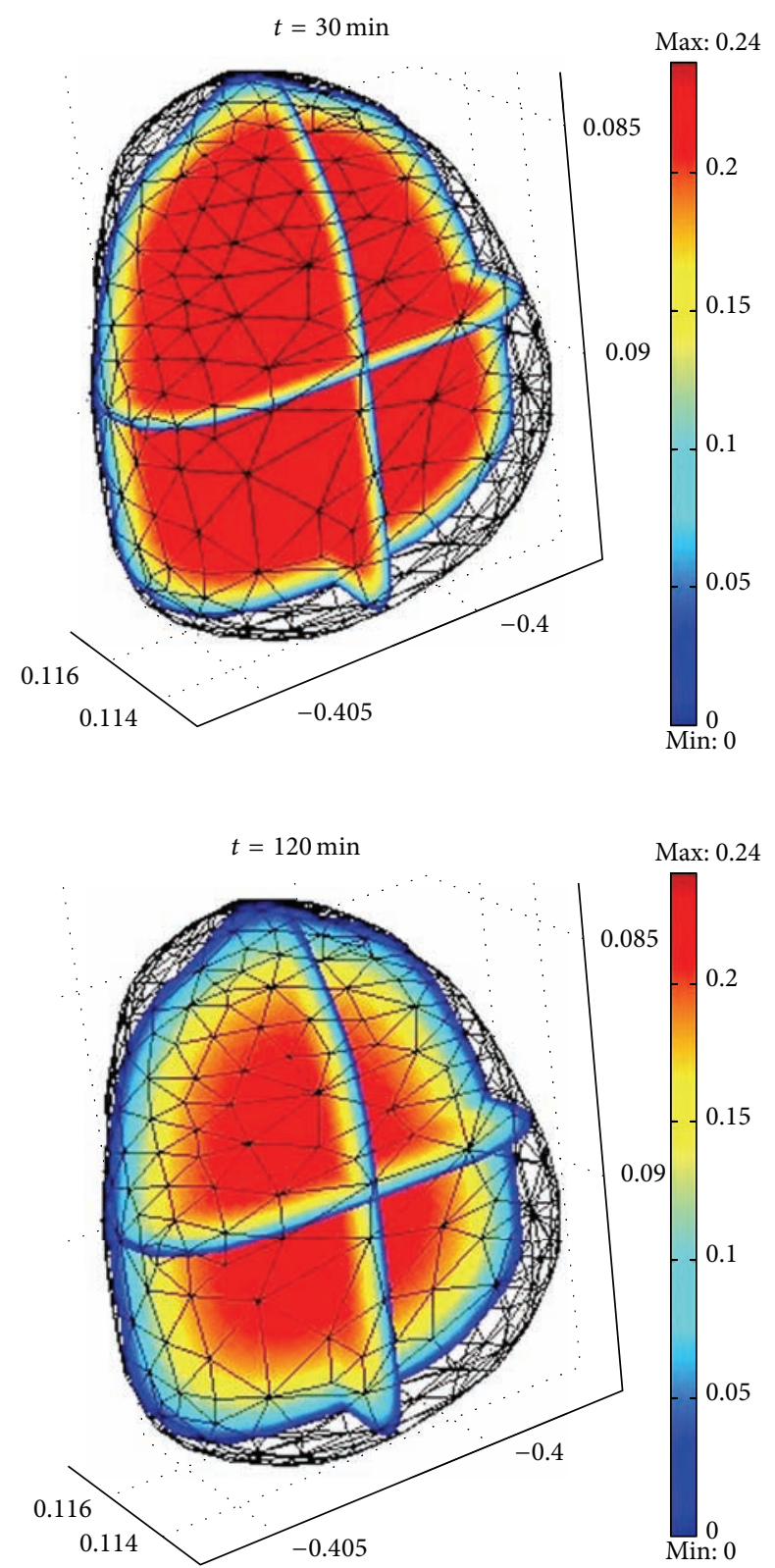

FIGURE 10: Slices of moisture distribution in the corn kernel at drying temperature $T_{1}=328 \mathrm{~K}$.

In selecting hot wind velocity, we should ensure that mass transfer outside the corn kernel takes place.

5.4. Energy Consumption Analysis. The effects of temperature and velocity of hot wind on energy consumption are visibly seen in Figures 18 and 19. Temperature $T_{3}=348 \mathrm{~K}$ and hot wind velocity $u_{1}=u_{0}$ account for the largest energy consumption in the drying simulations. The temperature and hot wind velocity have a linear relationship with energy consumption. Figure 20 shows that energy consumption changes along with diffused moisture. Given that wind velocity setting is needed only in considering mass transfer effects, the equilibrium moisture content should be maintained at a sufficiently low level. The air velocity can be appropriately reduced, thereby decreasing energy consumption. Heat transfer rapidly occurs and the drying of corn kernels is the process controlled by mass transfer. The choice of drying temperature depends primarily on the influence of mass transfer.

\section{Conclusion}

A 3D real body geometric model reconstruction of a single corn kernel is generated on the basis of CT with MIMICS and ANSYS. Based on the geometric model and a 3D drying mathematical model, the simulation calculation for the hot air drying of a corn kernel sample is 


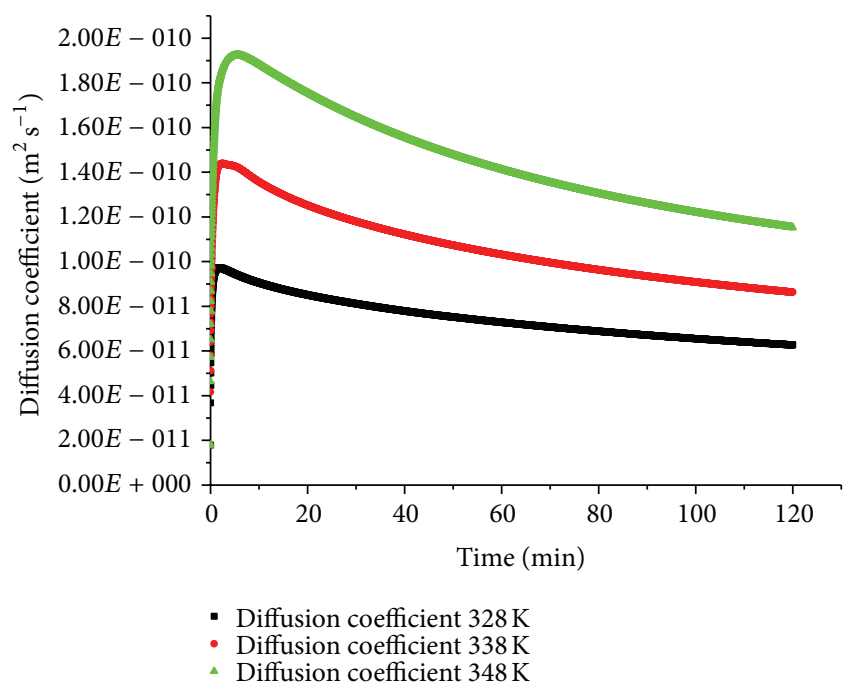

Figure 11: Average diffusion coefficient curves at different temperatures.

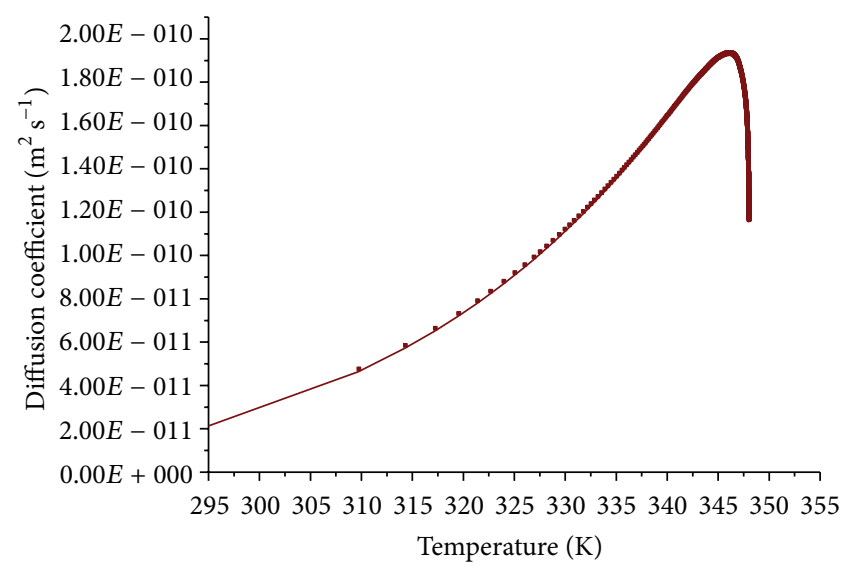

Figure 12: Diffusion coefficient via temperature at $T_{3}=348 \mathrm{~K}$.

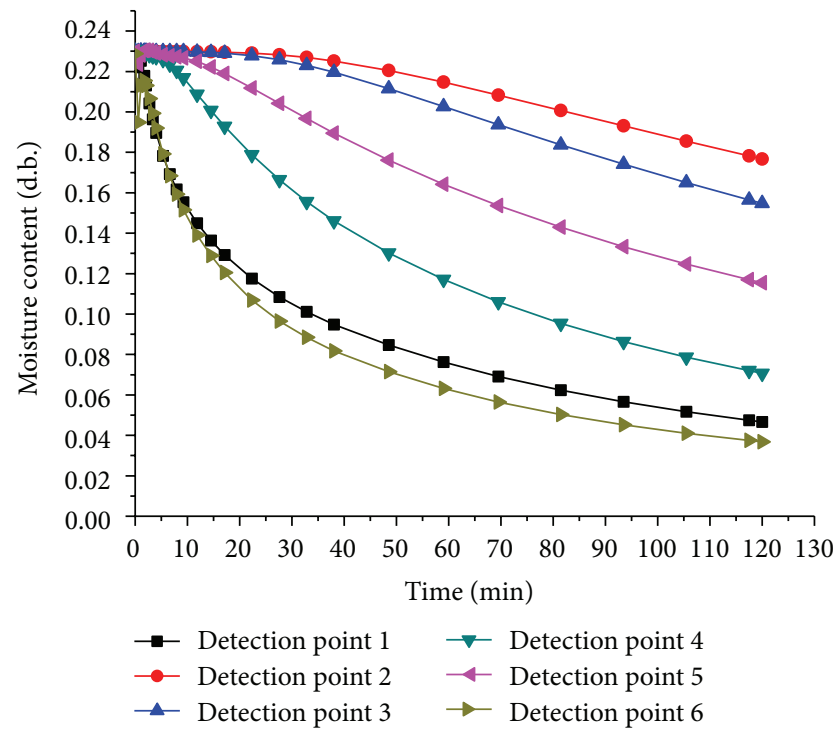

FIGURE 13: Moisture content curves at detection points when $T_{3}=$ $348 \mathrm{~K}$.

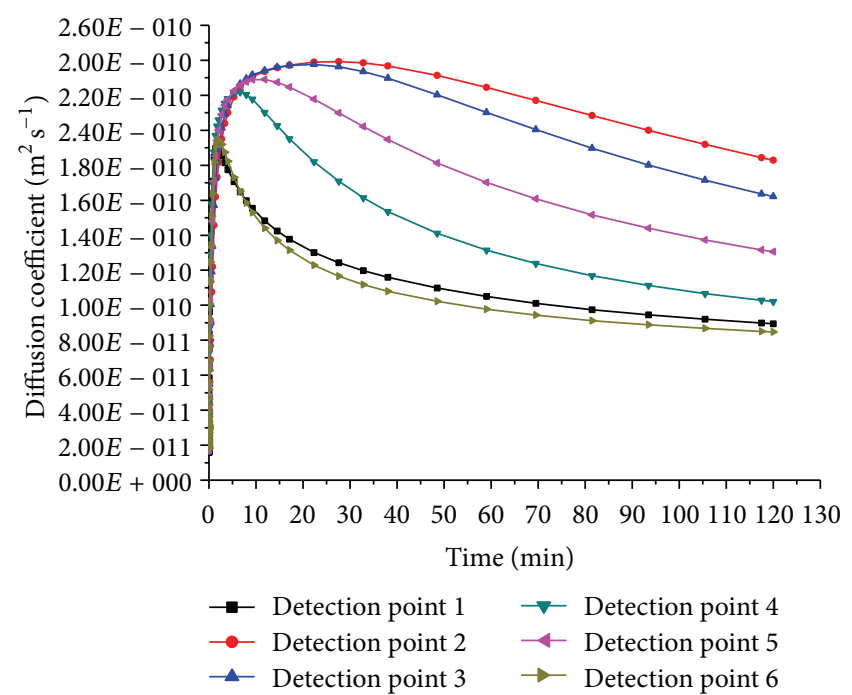

FIGURE 14: Diffusion coefficient curve of detection points at $T_{3}=$ $348 \mathrm{~K}$.

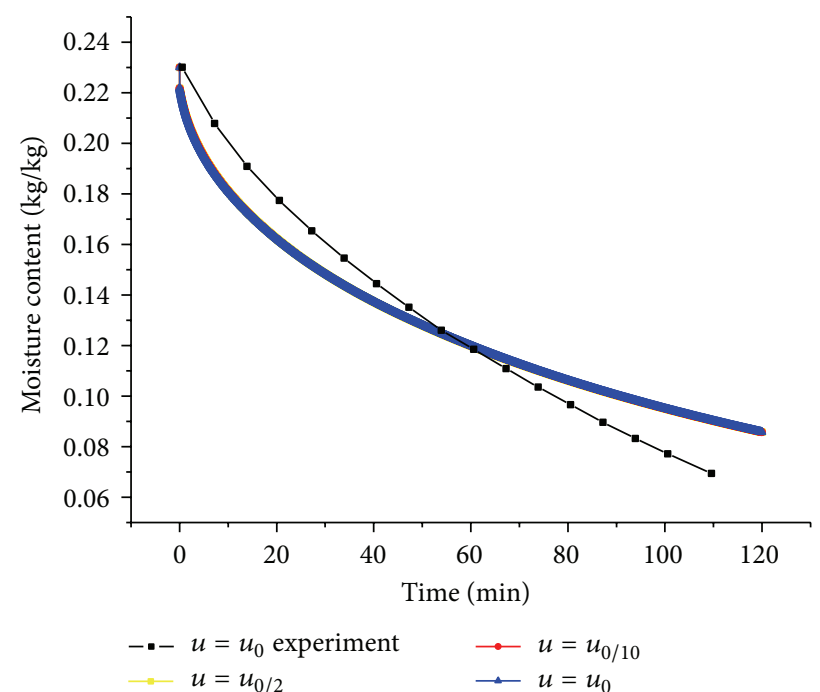

FIGURE 15: Drying curves at different hot wind velocities and the experiment drying curve at $u=u_{0}$.

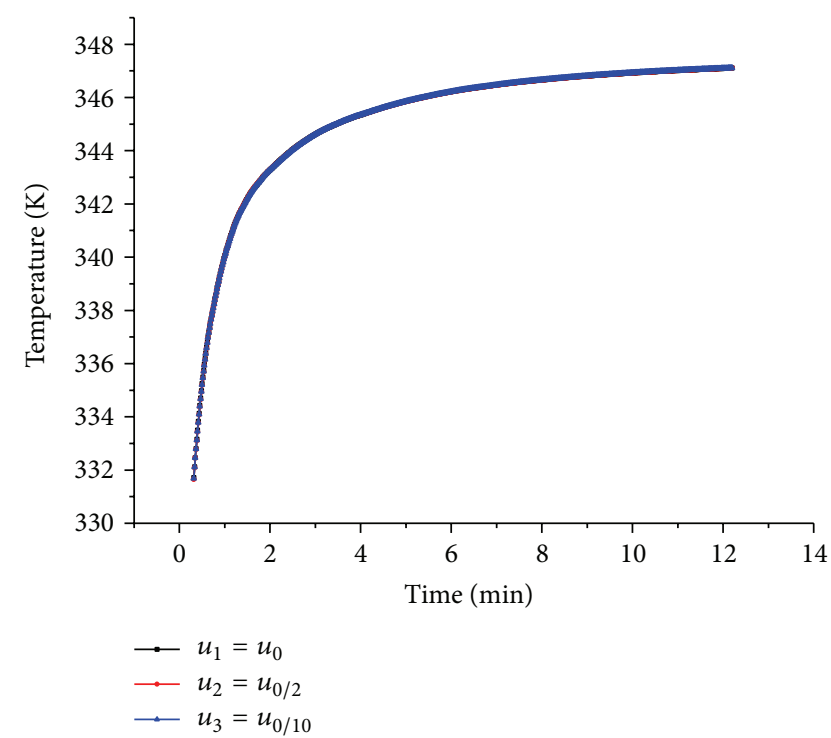

FIGURE 16: Temperature via different hot wind velocities. 


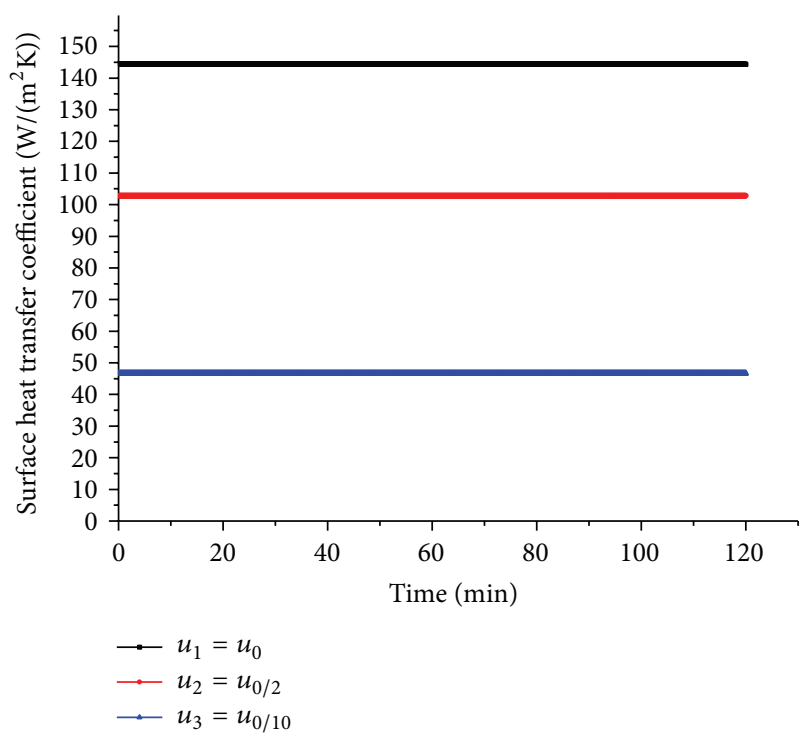

Figure 17: Comparison of surface heat transfer coefficients at different hot wind velocities.

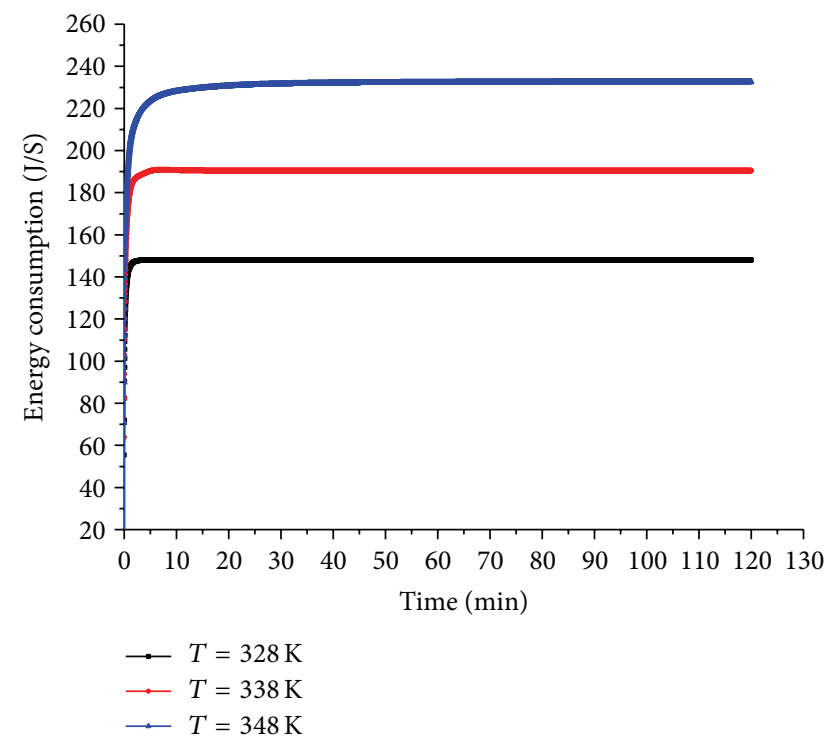

FIGURE 18: Different energy consumption curves at different drying temperatures.

carried out using COMSOL software. The results indicate that hot air drying is a process governed by interior mass transfer. Air velocity and temperature impose weak effects on heat transfer. Air temperature significantly affects the mass transfer process, whereas air velocity poses almost no effect. An essential requirement is that air velocity should ensure that external moisture diffuses from the corn surface to the ambient. The selection of air temperature and velocity depends primarily on mass transfer effects. A low velocity lessens energy consumption.

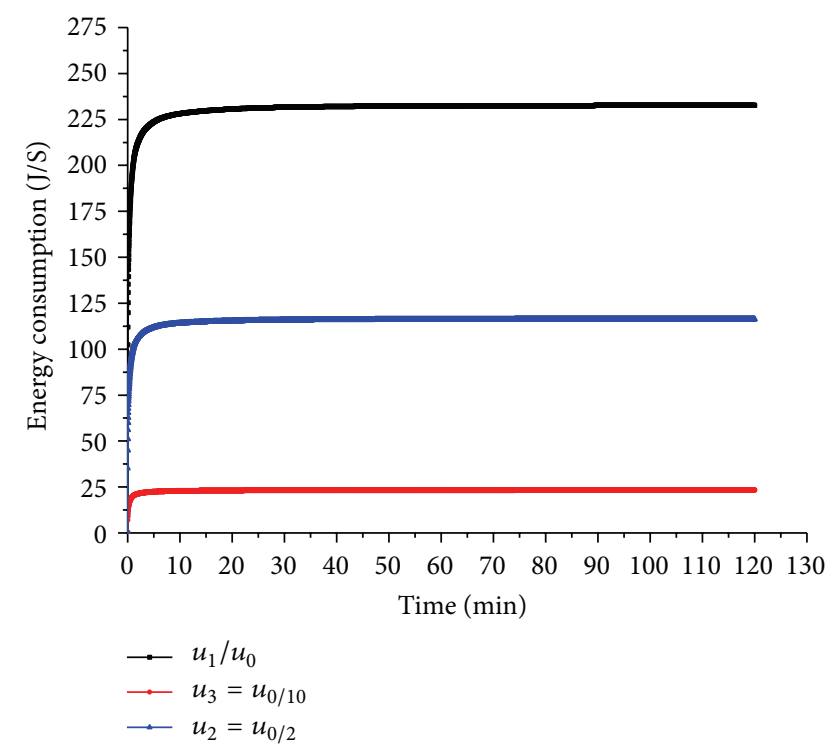

FIGURE 19: Different energy consumption curves at different hot wind velocities when $T_{3}=348 \mathrm{~K}$.

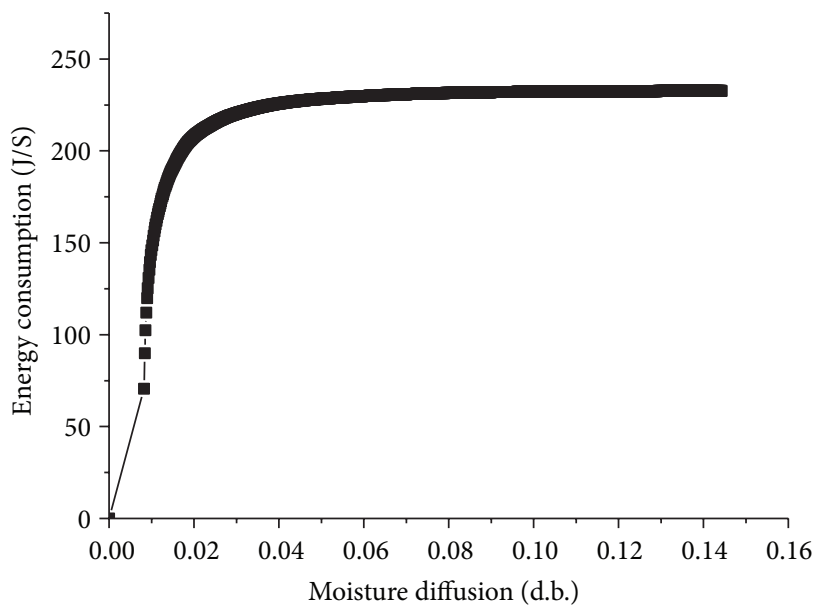

FIGURE 20: Energy consumption curves with diffused moisture at $T_{3}=348 \mathrm{~K}$; initial moisture content is $M_{0}=0.23$ (d.b.); wind speed $u=u_{0}$.

\section{Acknowledgment}

This research was supported by the National Natural Science Foundation of China Grants no. 51176027 and no. 31000665.

\section{References}

[1] J. Yongsawatdigul and S. Gunasekaran, "Microwave-vacuum drying of cranberries. Part I: energy use and efficiency," Journal of Food Processing and Preservation, vol. 20, no. 2, pp. 121-143, 1996.

[2] M. Aghbashlo, M. H. Kianmehr, and H. Samimi-Akhijahani, "Influence of drying conditions on the effective moisture diffusivity, energy of activation and energy consumption during the thin-layer drying of berberis fruit (Berberidaceae)," Energy Conversion and Management, vol. 49, no. 10, pp. 2865-2871, 2008. 
[3] D. R. Costa, A. F. de Lacerda Filho, J. de Sousa e Silva, D. Marçal de Queiroz, and J. P. Dadalto, "Energy consumption analysis of a system of drying of maize in mechanical dryer," Revista Engenharia na Agricultura, vol. 18, no. 2, pp. 151-164, 2010.

[4] R. J. Gustafson, D. R. Thompson, and S. Sokhansanj, “Temperature and stress analysis of Corn kernel-finite element analysis," Transactions of the American Society of Agricultural Engineers, vol. 22, no. 4, pp. 955-960, 1979.

[5] Y. Li and C. Cao, "Application of moisture diffusion model within an individual grain kernel," Transactions of the CSAE, vol. 9, no. 1, pp. 74-82, 1993.

[6] Z. Zhang and N. Kong, "Nonequilibrium thermal dynamic modeling of porous medium vacuum drying process," Mathematical Problems in Engineering, vol. 2012, Article ID 347598, 22 pages, 2012.

[7] C. Jia and C. Cao, "Process of tow-dimensional heat and mass transfer in corn kernel," Journal of Beijing Agriculture Engineering University, vol. 15, no. 1, pp. 45-51, 1995.

[8] M. Neményi, I. Czaba, A. Kovács, and T. Jáni, "Investigation of simultaneous heat and mass transfer within the maize kernels during drying," Computers and Electronics in Agriculture, vol. 26, no. 2, pp. 123-135, 2000.

[9] S. Zhang, J. Liu, N. H. Kong, and Z. J. Zhan, "Hot air drying simulation of corn grains based on 3D real body model," in Proceedings of the 18th International Drying Symposium, Xiamen, China, 2012.

[10] P. K. Ghosh, D. S. Jayas, E. A. Smith, M. L. H. Gruwel, N. D. G. White, and P. A. Zhilkin, "Mathematical modelling of wheat kernel drying with input from moisture movement studies using magnetic resonance imaging (MRI), Part I: model development and comparison with MRI observations," Biosystems Engineering, vol. 100, no. 3, pp. 389-400, 2008.

[11] J. H. Perez, F. Tanaka, and T. Uchino, "Modeling of mass transfer and initiation of hygroscopically induced cracks in rice grains in a thermally controlled soaking condition: with dependency of diffusion coefficient to moisture content and temperatureA 3D finite element approach," Journal of Food Engineering, vol. 111, no. 3, pp. 519-527, 2012.

[12] H. P. Song, S. R. Delwiche, and M. J. Line, "Moisture distribution in a mature soft wheat grain by three-dimensional magnetic resonance imaging," Journal of Cereal Science, vol. 27, no. 2, pp. 191-197, 1998.

[13] S. M. Goñi, E. Purlis, and V. O. Salvadori, "Three-dimensional reconstruction of irregular foodstuffs," Journal of Food Engineering, vol. 82, no. 4, pp. 536-547, 2007.

[14] C. Cao and W. Zhu, Computer Simulation of Drying Technology Process of Farm Products, Chinese Agriculture Press, Beijing, China, 2001.

[15] K. Haghighi and L. J. Segerlind, "Modeling simultaneous heat and mass transfer in anisotropic sphere-a finite element approach," Transactions of the American Society of Agricultural Engineers, vol. 31, no. 2, pp. 629-637, 1988.

[16] M. Parti, "Evaluation of selected mathematical models for grain drying," in Drying' 91, 1991. 


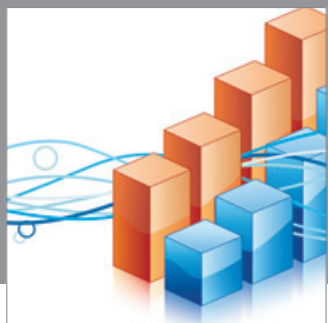

Advances in

Operations Research

mansans

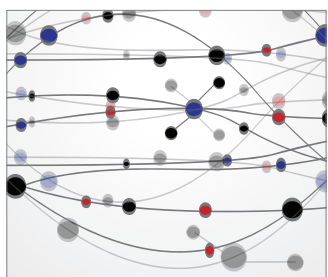

The Scientific World Journal
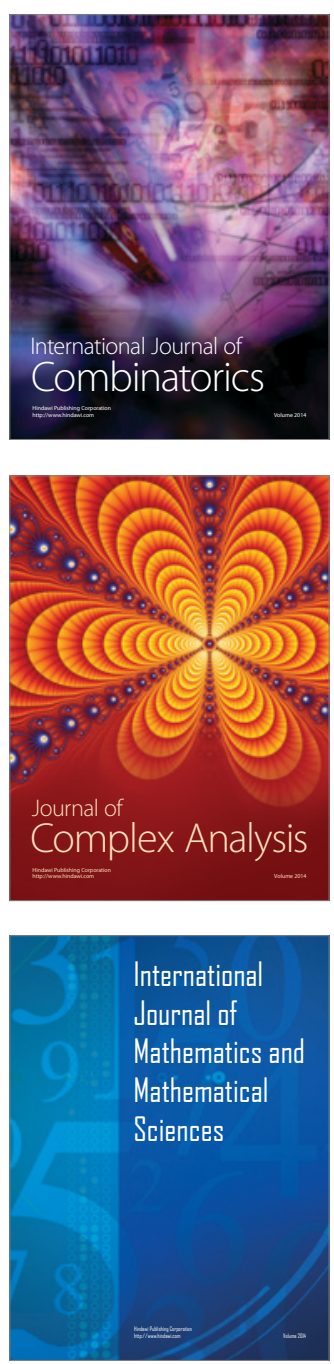
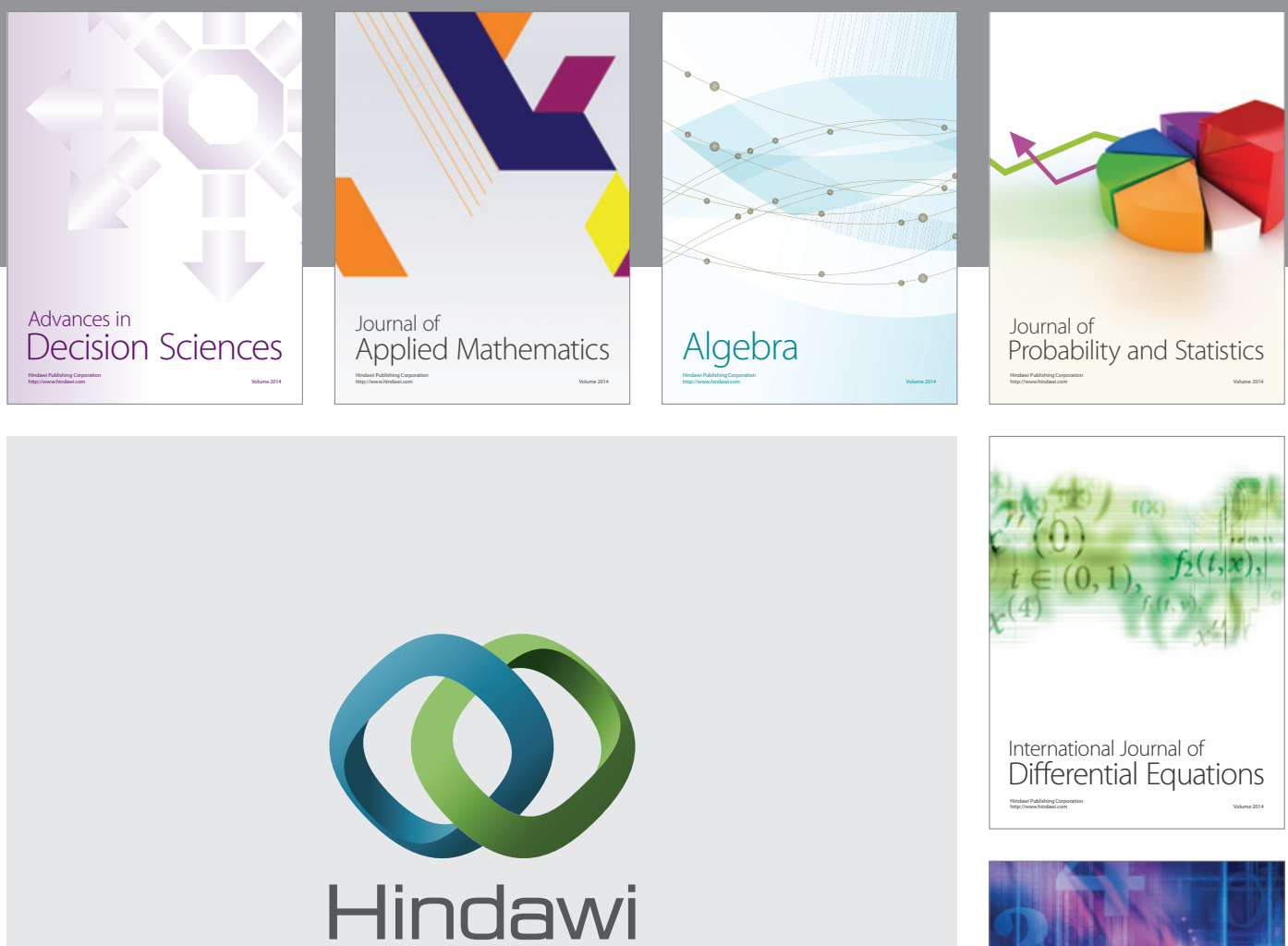

Submit your manuscripts at http://www.hindawi.com
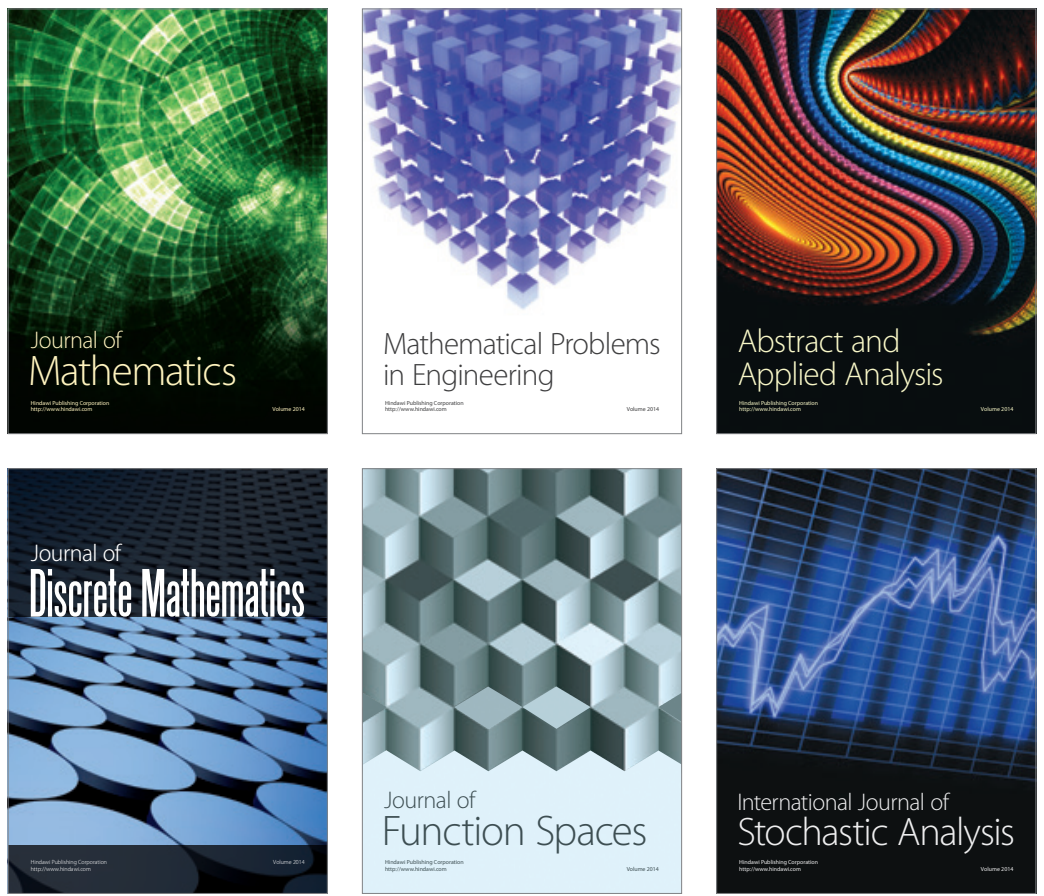

Journal of

Function Spaces

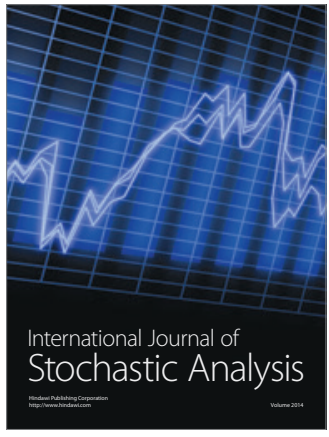

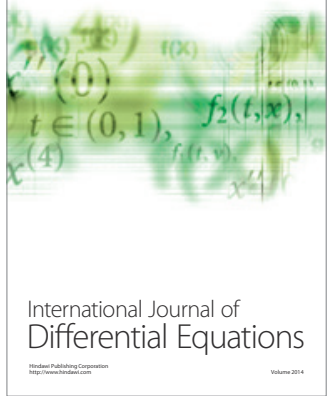
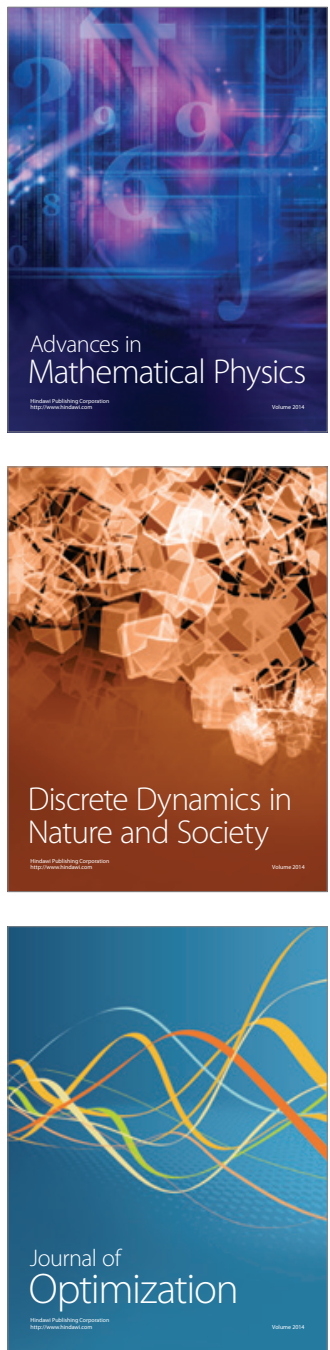